\title{
TrkB Signaling Is Required for Both the Induction and Maintenance of Tissue and Nerve Injury-Induced Persistent Pain
}

\author{
Xidao Wang, ${ }^{1}$ Joseline Ratnam, ${ }^{2}$ Bende Zou, ${ }^{2}$ Pamela M. England, ${ }^{2}$ and Allan I. Basbaum ${ }^{1}$ \\ ${ }^{1}$ Departments of Anatomy and Physiology and W. M. Keck Foundation Center for Integrative Neuroscience, and ${ }^{2}$ Department of Pharmaceutical Chemistry, \\ University of California, San Francisco, San Francisco, California 94158
}

\begin{abstract}
Activation of primary afferent nociceptors produces acute, short-lived pain, and tissue or nerve injury induces long-term enhancement of nociceptive processing, manifested as hypersensitivity to thermal and mechanical stimulation. Here we used a chemical-genetic and pharmacological approach to study the contribution of the receptor tyrosine kinase, type 2 (TrkB) to the generation and maintenance of injury-induced persistent pain. We performed the studies in wild-type mice and transgenic (TrkB $\left.{ }^{\mathrm{F} 616 \mathrm{~A}}\right)$ mice that express mutant but fully functional TrkB receptors. By injecting a small molecule derivative of the protein kinase inhibitor protein phosphatase 1 (1NM-PP1), it is possible to produce highly selective inhibition of TrkB autophosphorylation in adult mice, without interfering with the activity of other protein kinases. We report that oral administration of 1NM-PP1, at doses that blocked phosphorylation of TrkB in the spinal cord, had no effect in behavioral tests of acute heat, mechanical, or chemical pain sensitivity. However, the same pretreatment with 1NM-PP1 prevented the development of tissue- or nerve injury-induced heat and mechanical hypersensitivity. Established hypersensitivity was transiently reversed by intraperitoneal injection of 1NM-PP1. Although interfering with TrkB signaling altered neither acute capsaicin nor formalin-induced pain behavior, the prolonged mechanical hypersensitivity produced by these chemical injuries was prevented by 1NM-PP1 inhibition of TrkB signaling. We conclude that TrkB signaling is not only an important contributor to the induction of heat and mechanical hypersensitivity produced by tissue or nerve injury but also to the persistence of the pain.
\end{abstract}

\section{Introduction}

That brain-derived neurotrophic factor (BDNF) contributes to the processing of nociceptive messages was originally suggested by a dramatic alteration in the levels of BDNF expression in the spinal cord after tissue or nerve injury (Ernfors et al., 1993; Michael et al., 1997; Cho et al., 1998; Zhou et al., 1999; Fukuoka et al., 2001; Pezet et al., 2002; Merighi et al., 2008b). BDNF levels increased in peptidergic nociceptors, and there is de novo expression in cell bodies of large-diameter afferents (Cho et al., 1998; Mannion et al., 1999; Michael et al., 1999). Experimental manipulation of either BDNF or the receptor tyrosine kinase, type 2 (TrkB), the receptor through which BDNF signals, also significantly alters nociceptive processing. For example, genetic deletion of BDNF from $\mathrm{Na}_{\mathrm{V}} 1.8$-expressing nociceptors decreased inflammation-induced thermal hyperalgesia (Zhao et al., 2006). Surprisingly, acute thermal pain responsiveness increased. In contrast, intrathecal administration of antisense directed against either BDNF or TrkB mRNA decreased both acute heat pain and inflammation-associated thermal hyperalgesia (Groth and Aanon-

Received Sept. 8, 2008; revised Feb. 3, 2009; accepted March 20, 2009.

This work was supported by National Institutes of Health Grants NS48499 and 14627. The TrkB ${ }^{\mathrm{F} 616 \mathrm{~A}}$ mice were kindly provided by Dr. David Ginty at Johns Hopkins School of Medicine and with permission of the Campus Common Gateway Interface (CGI) Facility and Princeton.

Correspondence should be addressed to Allan I. Basbaum, Department of Anatomy, University of California, San Francisco, 1550 4th Street, San Francisco, CA 94158. E-mail: allan.basbaum@ucsf.edu.

D01:10.1523/JNEUROSCI.4288-08.2009

Copyright $\odot 2009$ Society for Neuroscience $\quad$ 0270-6474/09/295508-08\$15.00/0 sen, 2002), as did reduction of BDNF via intrathecal administration of anti-BDNF antibodies or by sequestration of BDNF via intrathecal TrkB-IgG (Mannion et al., 1999; Yajima et al., 2002).

With respect to the processing of mechanical pain, some studies reported no reduction of the mechanical hypersensitivity induced by partial sciatic nerve ligation (Zhao et al., 2006) and no change in capsaicin-induced secondary mechanical hyperalgesia after sequestration of BDNF (Mannion et al., 1999). Other studies, however, found that sequestration of BDNF (Marcol et al., 2007) or small interfering RNA-mediated reduction of microglial BDNF synthesis significantly reduced nerve injury-induced mechanical allodynia (Coull et al., 2005). In contrast, intrathecal injection of BDNF reduced the hyperalgesia produced by partial nerve injury (Lever et al., 2003). Finally, although Kerr et al. (1999) found little effect of reducing BDNF levels, TrkB-IgG sequestration of BDNF decreased the hypersensitivity produced by repeated tactile stimulation in the setting of inflammation (Mannion et al., 1999).

These differences may reflect the locus and/or the magnitude of the BDNF reduction produced by the various approaches. The genetic deletion experiments (Zhao et al., 2006) indicate that peptidergic afferents are the source of BDNF that is critical to heat pain processing. In contrast, the locus of action after intrathecal administration cannot be unequivocally established. BNDF levels in primary afferents, postsynaptic neurons, and/or microglia could be relevant. Most importantly, because TrkB is not the only route through which BDNF signals (Huang and Reichardt, 2003; 
Fan et al., 2008), studies directed at the selective contribution of TrkB signaling are required.

Here we used a transgenic mouse $\left(\operatorname{TrkB}{ }^{\mathrm{F} 616 \mathrm{~A}}\right)$ that expresses a mutated TrkB (Chen et al., 2005). By administering an antagonist, 1NM-PP1 (a small molecule derivative of the protein kinase inhibitor protein phosphatase 1), that selectively binds to the mutated receptor, it is possible to produce a profound and reversible blockade of TrkB autophosphorylation (Wang et al., 2003; Chen et al., 2005; Kaneko et al., 2008). Using this integrated chemical-genetic approach, we provide evidence that TrkB signaling is critical for both the induction and maintenance of the heat and mechanical hypersensitivity produced by tissue or nerve injury.

\section{Materials and Methods}

Generation of $\operatorname{Trk} B^{F 616 A}$ mice. TrkB ${ }^{\mathrm{F} 616 \mathrm{~A}}$ knock-in mice were generated as described previously (Chen et al., 2005). Heterozygous TrkB ${ }^{\text {F616A }}$ mice were used to generate homozygous $\operatorname{TrkB}{ }^{\text {F616A }}$ mice. Wild-type (WT) littermates served as controls.

Chemical synthesis of 1NM-PP1 and drug delivery. 1NM-PP1 was synthesized as described previously (Wang et al., 2003) and was administered in the drinking water $(5.0 \mu \mathrm{M}$ 1NM-PP1). For systemic administration, we made an intraperitoneal injection of 5.0 $\mu \mathrm{M}$ 1NM-PP1 in saline (10 $\mu \mathrm{l} / \mathrm{g}$, i.p.) (Wang et al., 2003).

Western immunoblots. The following primary antibodies were used: anti-pan Trk (C-14) polyclonal antibody (sc-139) and anti-phospho-Trk (E6) monoclonal antibody (sc-8058) were from Santa Cruz Biotechnology. Anti-TrkB monoclonal antibody was from BD Biosciences (catalog \#610102), and anti-TrkA polyclonal was from Upstate (catalog \#06-574). The following secondary antibodies were used: horseradish peroxidaseconjugated anti-rabbit and anti-mouse antibodies were from Jackson ImmunoResearch.

Immunoprecipitation and Western blots. Spinal cords were removed quickly by hydraulic extrusion with saline containing $1.0 \mathrm{~mm}$ sodium orthovanadate and flash frozen in liquid nitrogen. Cords were then homogenized in $500 \mu \mathrm{l}$ of radioimmunoprecipitation assay (RIPA) buffer [1\% NP-40, 1\% deoxycholic acid, 0.1\% SDS, 20 mM HEPES, pH 7.4, 100 mм NaCl, 1 mm EGTA, 1 mm sodium orthovanadate, $50 \mathrm{~mm} \mathrm{NaF}, 1.0 \mathrm{~mm}$ PMSF, $20 \mathrm{~mm}$ b-glycerophosphate, and Complete EDTA-free protease inhibitor cocktail (Roche)] and incubated for $30 \mathrm{~min}$ on ice. All subsequent steps were performed at $4^{\circ} \mathrm{C}$. Lysates were centrifuged at 16,000 $\times$ $g$ for $15 \mathrm{~min}$, and equal quantities of the supernatants were incubated with TrkA antibody for $4 \mathrm{~h}$, followed by Protein A/G agarose for $4 \mathrm{~h}$ to preclear the samples of TrkA. Preliminary experiments showed that TrkA was completely removed in the first round of immunoprecipitation. The supernatant was then incubated with pan-Trk antibody overnight, followed by addition of Protein A/G agarose for $4 \mathrm{~h}$. The resultant immunoprecipitates were washed three times with RIPA and one time with TBS containing $1.0 \mathrm{~mm}$ PMSF plus $1.0 \mathrm{~mm}$ sodium orthovanadate and extracted by boiling in $30 \mu \mathrm{l}$ of SDS sample buffer. Proteins were resolved by SDS-PAGE, transferred to polyvinylidene difluoride membranes, and probed with anti-phospho-Trk antibodies overnight. SuperSignal Westfemto substrate (Pierce) was used to detect phospho-Trk by ECL. Blots were then stripped and reprobed with anti-Trk $\mathrm{B}^{\mathrm{F} 616 \mathrm{~A}}$ antibodies. Densitometric analysis of bands was performed using NIH Image 1.62 software. To correct for variations in loading, the data are expressed as percentage phospho-Trk of total TrkB ${ }^{\text {F616A }}$ detected in each lane.

Behavior. Experiments were performed following the guidelines of the International Association for the Study of Pain and with approval of the University of California, San Francisco Institutional Animal Care and Use Committee. All experiments were performed blind to the genotype of the mice. Mice of both sexes (20-35 g) were housed in a $12 \mathrm{~h}$ light/dark cycle at $21^{\circ} \mathrm{C}$. Comparisons were made between $\operatorname{TrkB}{ }^{\mathrm{F} 616 \mathrm{~A}}$ mice and their wild-type littermates. Complete details of most of the pain models used [von Frey testing of mechanical sensitivity, Hargreaves radiant heat test of thermal sensitivity, formalin test, Complete Freund's adjuvant (CFA)-induced chronic inflammation and sciatic nerve injury (SNI) model of neuropathic pain] can be found in the study of Shields et al. (2007). Methods for testing after formalin mechanical hypersensitivity can be found in the study of Zeitz et al. (2004). An individual blind to genotype performed all behavioral experiments.

Capsaicin-induced licking and postinjection mechanical hyperalgesia. Mice were injected with $3.0 \mu \mathrm{g}$ of capsaicin in $10 \mu \mathrm{l}$ of $10 \%$ ethanol/10\% Tween/80\% saline into the ankle. Thermal and mechanical sensitivity were tested as above. Postcapsaicin-induced mechanical hypersensitivity was tested as for postformalin hypersensitivity (Zeitz et al., 2004).

Statistical analyses. All data are presented as mean \pm SEM. Data were analyzed using two- or one-way repeated measures ANOVA with Tukey's post hoc analysis. $p<0.05$ was regarded as significant.

\section{Results \\ Inhibition of TrkB signaling does not alter mechanical or thermal nociceptive thresholds}

Previous studies reported that PP1 derivatives, including 1NM$\mathrm{PP} 1$, potently and selectively inhibited mutant $\operatorname{TrkB}{ }^{\mathrm{F} 616 \mathrm{~A}}$ receptors. The $\mathrm{IC}_{50}$ for $1 \mathrm{NM}-\mathrm{PP} 1$ inhibition of TrkB autophosphorylation was $\sim 3.0 \mathrm{~nm}$. Importantly, there was no inhibition of wildtype TrkA or TrkB with drug concentration as high as $10 \mu \mathrm{M}$, and, even at these high doses, there are no adverse side effects. Here we measured baseline thermal and mechanical nociceptive thresholds in wild-type and $\operatorname{Tr} k \mathrm{~B}^{\mathrm{F} 616 \mathrm{~A}}$ knock-in mice, before and after allowing the mice ad libitum access to drinking water containing $5.0 \mu \mathrm{M} 1 \mathrm{NM}-\mathrm{PP} 1$. We found no significant change of mechanical (von Frey test) or thermal (Hargreaves test) nociceptive thresholds in either wild-type or $\operatorname{TrkB}^{\mathrm{F} 616 \mathrm{~A}}$ knock-in mice (data not shown). We conclude that TrkB phosphorylation is not required for acute nociceptive processing.

\section{Inhibition of TrkB signaling prevents capsaicin-induced mechanical hyperalgesia}

Although many in vitro studies established that 1NM-PP1 completely prevents TrkB phosphorylation, there is much less evidence that effective blockade could be produced in vivo. Here we validated the approach in a model of chemically evoked persistent pain, using oral doses reported previously to block phosphorylation in vivo. In these studies, we used an algogen, capsaicin, that maximally stimulates nociceptors that express TRPV1. In the mouse, the great majority of TRPV1-expressing nociceptors express BDNF and the neuropeptides substance $\mathrm{P}$ and calcitonin gene-related peptide. Capsaicin-induced activation of TRPV1-expressing nociceptors not only evokes pain behavior (shaking and licking of the injected hindpaw) but also produces a profound secondary hyperalgesia, namely, increased mechanical sensitivity outside of the area of injury (Sun et al., 2006; Walker et al., 2007). The hyperalgesia is presumed to arise from a central sensitization of spinal cord dorsal horn circuits secondary to the intense afferent discharge induced by capsaicin. By administering $1 \mathrm{NM}-\mathrm{PP} 1$ in the drinking water, we could test the hypothesis that the central sensitization requires TrkB phosphorylation. To confirm that there was inhibition of TrkB phosphorylation, we collected lumbar spinal cords from a parallel group of mice treated under the same conditions. Finally, to confirm that TrkB phosphorylation occurs normally in the mice, we also studied capsaicin-treated wild-type and $\operatorname{TrkB}{ }^{\mathrm{F} 616 \mathrm{~A}}$ knock-in mice that did not receive 1NM-PP1.

Consistent with the lack of effect of blocking TrkB signaling on acute heat or mechanical thresholds, we found no effect of 1NM-PP1 on the magnitude of the pain behavior produced by capsaicin. Figure $1 \mathrm{~A}$ further illustrates that injection of capsaicin $(3.0 \mu \mathrm{g} / 10 \mu \mathrm{l})$ into the ankle of either wild-type mice or transgenic mice that did not receive $1 \mathrm{NM}-\mathrm{PP} 1$ induced a dramatic 
secondary mechanical hyperalgesia, manifested as a lowering of the mechanical pain threshold (tested on the plantar surface of the hindpaw). In addition, when we tested $\operatorname{TrkB}{ }^{\mathrm{F} 616 \mathrm{~A}}$ knock-in mice treated with $5.0 \mu \mathrm{M}$ 1NM-PP1 in the drinking water for 2-3 d before the injection of capsaicin, we found no change of the mechanical threshold (tested at 15, 30, or $60 \mathrm{~min}$ after capsaicin). The capsaicin treatment resulted in an expected profound increase in thermal response latencies, and this was found in both control and 1NM-PP1treated mice (Fig. 1B). The reduced responsiveness to noxious heat presumably results either from desensitization of the TRPV1 channel, and thus cross desensitization to heat, or it may have occurred secondary to TRPV1-mediated destruction of the peripheral terminals of these afferents.

Binding of BDNF to TrkB leads to dimerization of the receptor and its autophosphorylation on tyrosine residues (Lindsay, 1996). To determine whether oral 1NM-PP1 inhibits TrkB ${ }^{\mathrm{F} 616 \mathrm{~A}}$ phosphorylation in the capsaicin model, we repeated the capsaicin experiment, in this case, under pentobarbital anesthesia. Five minutes after ankle injection of capsaicin, lumbar spinal cords were collected and flash frozen. Trk receptors were immunoprecipitated, and the relative amounts of phospho-Trk and total TrkB ${ }^{\mathrm{F} 616 \mathrm{~A}}$ were assessed.

Western blots showed that capsaicin-induced TrkB phosphorylation in the wild-type and $\operatorname{TrkB}{ }^{\mathrm{F} 616 \mathrm{~A}}$ mice was comparable in the absence of 1NM-PP1 (Fig. 1C) (phospho-Trk/total TrkB, $63.3 \pm 1.8 \%$ WT vs $\left.67.0 \pm 1.7 \% \operatorname{TrkB}{ }^{\mathrm{F} 616 \mathrm{~A}}\right)$. In contrast, blots of lumbar cords showed a marked increase of TrkB phosphorylation in the wild-type mice but not in the $T r k \mathrm{~B}^{\mathrm{F} 616 \mathrm{~A}}$ mice that received 1NM-PP1 (Fig. 1D) (phospho-Trk/total TrkB, $47.3 \pm$ $3.7 \%$ WT vs $\left.5.1 \pm 4.9 \% \operatorname{TrkB}^{\mathrm{F} 616 \mathrm{~A}}\right)$. Importantly, the total amount of TrkB loaded into the control and 1NM-PP1 lanes was comparable (Fig. 1C,D). Based on these results, we conclude that 1NM-PP1 significantly inhibited capsaicin-induced phosphorylation of TrkB. The concurrent loss of capsaicin-induced secondary mechanical hyperalgesia suggests that TrkB signaling underlies the sensitization that is necessary for the development of the mechanical hypersensitivity.

\section{Inhibition of TrkB signaling prevents and can reverse CFA- inflammation associated mechanical and thermal hyperalgesia}

With the ability to regulate TrkB signaling in the adult, we next asked whether TrkB phosphorylation is an important contributor in other models of injury-induced persistent pain. Hindpaw injection of CFA produces a profound mechanical and thermal hyperalgesia that peaks at approximately $3 \mathrm{~d}$ after its injection and persists for many days. In these studies, we allowed the mice to drink water containing 5.0 $\mu \mathrm{M}$ 1NM-PP1 for $2 \mathrm{~d}$ before making an intraplantar injection of CFA into the left hindpaw. Figure 2 shows that CFA-induced mechanical and heat hyperalgesia were both significantly reduced (prevented) in the $\operatorname{Trk} \mathrm{B}^{\mathrm{F} 616 \mathrm{~A}}$ mice treated with 1NM-PP1. Importantly, there was no effect on paw-
B Radiant heat
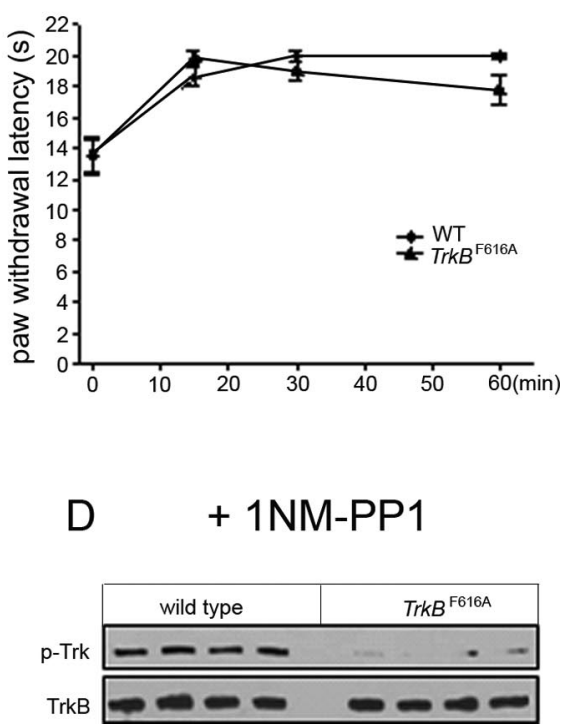

Figure 1. Inhibition of TrkB signaling by 1NM-PP1 in TrkB ${ }^{\mathrm{F} 616 \mathrm{~A}}$ mice prevents capsaicin-induced mechanical hyperalgesia and (3.0 latency to noxious heat did not differ between WT and TrkB ${ }^{\mathrm{F} 616 \mathrm{~A}}$ mice. C, D, Western blots of lumbar spinal cords from four WT and our TrkB ${ }^{\mathrm{F} 16 \mathrm{~A}}$ mice show that Trk phosphorylation after capsaicin treatment in the absence of 1NM-PP1 (C) in the drinking wate did not differ. Total TrkB loaded for WT and mutant mice is equivalent. In contrast, in the presence of 1NM-PP1 (D), there is a significant decrease of Trk phosphorylation in the $T r k B^{\mathrm{F} 616 \mathrm{~A}}$ mice (for quantification, see Results).

withdrawal thresholds contralateral to the CFA injection and no effect on the hypersensitivity induced in wild-type mice treated with 1NM-PP1.

To study the reversibility of the 1NM-PP1 effects, we next asked whether switching the mice to normal drinking water would reinstate the CFA-induced behavioral changes. Two days after drinking 1NM-PP1-free water, we observed that the mechanical and thermal hyperalgesia reappeared and were equivalent to the thresholds recorded in untreated TrkB ${ }^{\mathrm{F} 616 \mathrm{~A}}$ mice (Fig. $2 A, B)$. These studies demonstrate that TrkB signaling contributes to the development of CFA-induced hyperalgesia and suggest that there is a prolonged and possibly sustained increase of TrkB signaling as the inflammation persists.

To test the latter possibility more directly, we next asked whether blocking TrkB signaling could reverse previously established CFA-induced hyperalgesia. In these experiments, we followed the protocol of Kaneko et al. (2008) who showed that an intraperitoneal injection of 1NM-PP1 transiently blocked TrkB phosphorylation. We injected CFA into the hindpaw of mice and documented the decreased heat and mechanical thresholds. Three days later, we injected the mice with 1NM-PP1 (16.6 ng/g, i.p.) and measured withdrawal thresholds. Figure 3 illustrates that administration of 1NM-PP1 (intraperitoneal) produced a significant reversal of previously established mechanical and thermal hyperalgesia. The reversal was transient; the hyperalgesia returned after $90 \mathrm{~min}$ (Fig. $3 \mathrm{~A}, \mathrm{~B}$ ). The same treatment was without effect in wild-type mice. These results demonstrate that TrkB phosphorylation not only contributes to the induction of inflammation associated mechanical and thermal hyperalgesia but also is required for its persistence. In light of this demonstration of the important contribution of TrkB signaling, it is of interest that sequestering NGF with TrkA-IgG antibodies reduced the main- 
A

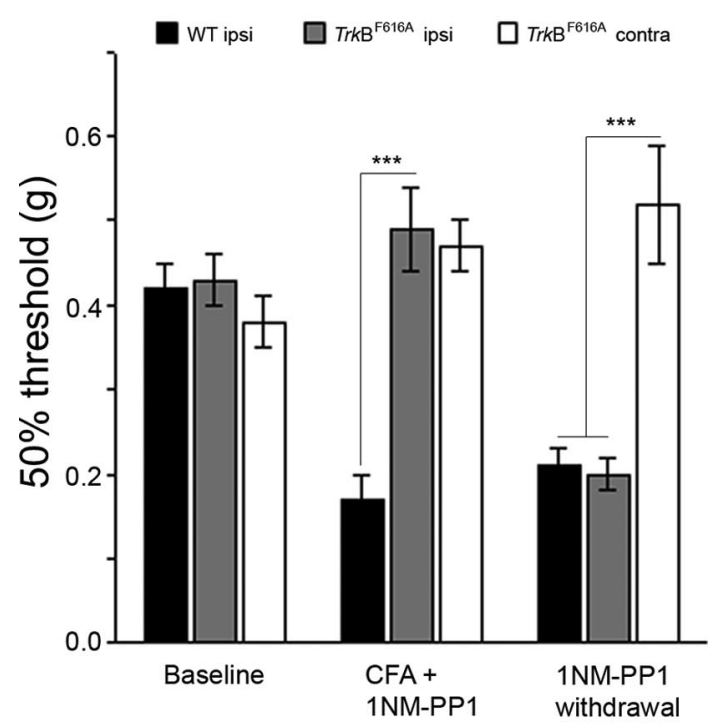

B

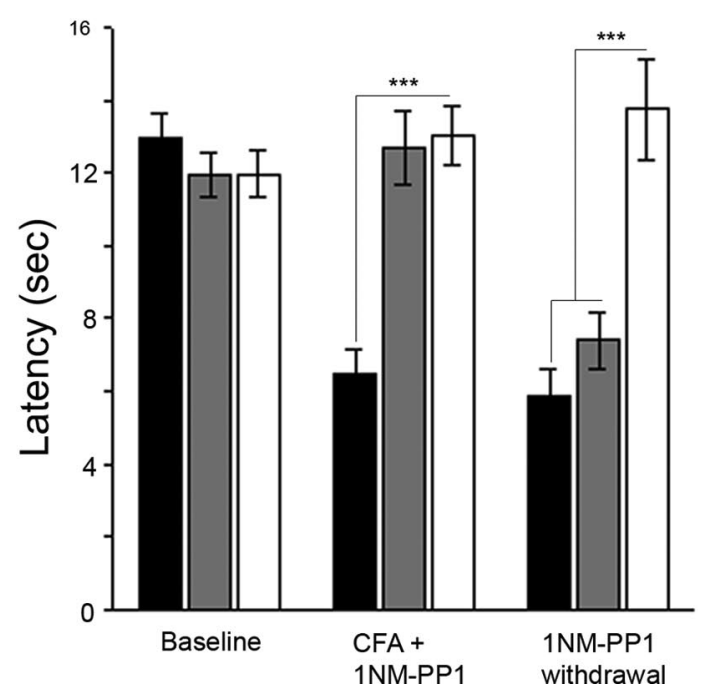

Figure 2. Inhibition of TrkB signaling prevents CFA-induced mechanical and heat hyperalgesia. 1NM-PP1 was added to the drinking water (final concentration, $5.0 \mu \mathrm{M}$ ), and mice had unlimited access to the water for 2-3 $d$ before and for $2 d$ after injection of CFA into the hindpaw. Baseline mechanical $(\boldsymbol{A})$ and heat ( $\boldsymbol{B}$; latency) withdrawal thresholds of the hindpaw did not differ in WT $(n=5)$ and $\operatorname{TrkB}^{\mathrm{F} 616 \mathrm{~A}}(n=5)$ mice. Two days after CFA, there was a significant drop in mechanical $(\boldsymbol{A})$ and heat $(\boldsymbol{B})$ thresholds in WT mice (black bars) compared with the thresholds in TrkB ${ }^{\mathrm{F} 616 \mathrm{~A}}$ mice (gray bars) or in the hindpaw contralateral to the CFAinjected paw of TrkB ${ }^{\text {F616A }}$ mice (white bars; ${ }^{* * *} p<0.001$ ). Two days after the 1NM-PP1 was withdrawn from the drinking water, the mechanical and heat thresholds of the CFA-injected hindpaw dropped significantly to that observed in WT mice and significantly different from the threshold of the contralateral paw of the $\operatorname{TrkB}{ }^{\mathrm{F} 616 \mathrm{~A}}$ mice $\left({ }^{* * *} p<0.001\right)$. ipsi, Ipsilateral; contra, contralateral.

tenance of a non-malignant bone injury pain but could not prevent its onset (Jimenez-Andrade et al., 2007). Together, these results highlight the differential contribution of TrkA and TrkB signaling to the development and maintenance of inflammatory pains.

TrkB signaling is required for the induction and maintenance of peripheral nerve injury-induced mechanical hyperalgesia Neuropathic pain represents a heightened pain sensitivity induced by peripheral nerve injury. In these conditions, normally
A von Frey test

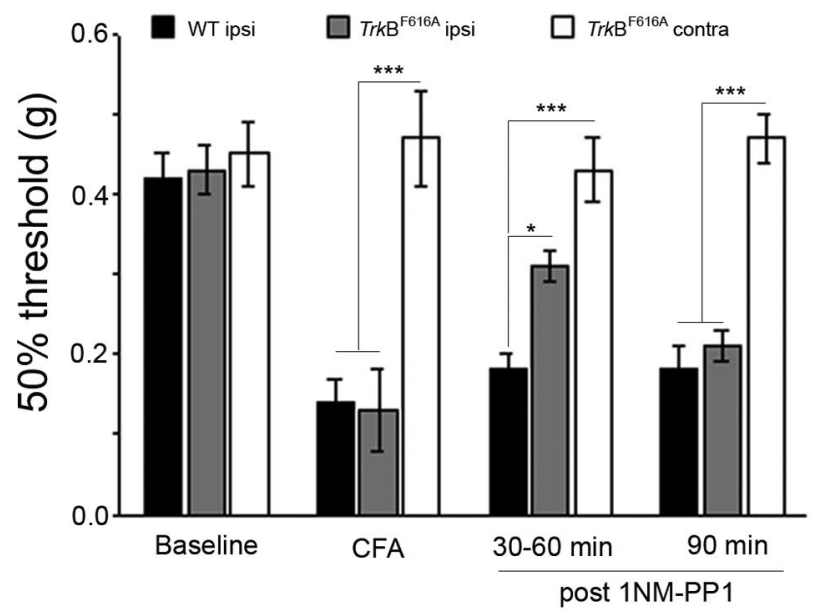

B

\section{Radiant heat}

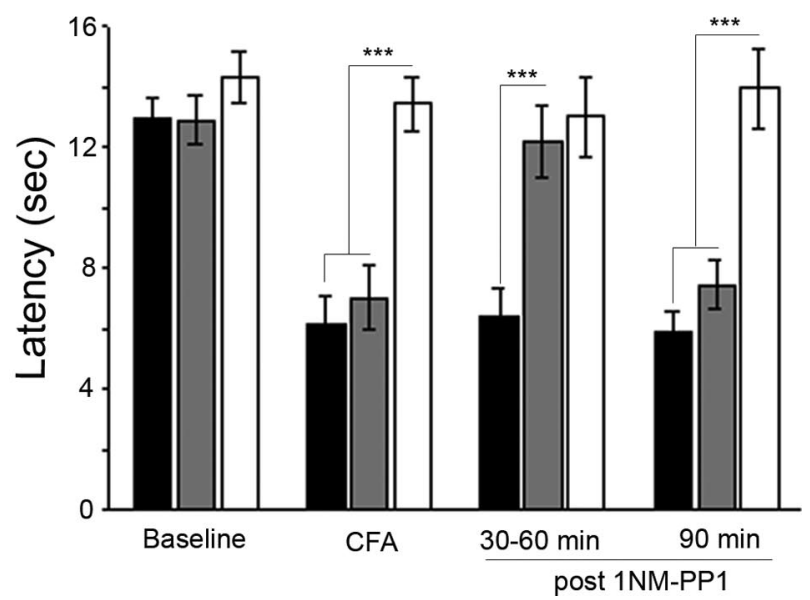

Figure 3. Inhibition of TrkB signaling can reverse established (FA-induced mechanical and heat hyperalgesia. Changes in mechanical $(\boldsymbol{A})$ and thermal $(\boldsymbol{B})$ threshold were measured at different time points after intraperitoneal injection of 1NM-PP1 $(16.6 \mathrm{ng} / \mathrm{g})$ in CFA-treated wild-type $(n=5)$ or $\operatorname{TrkB}^{\mathrm{F} 616 \mathrm{~A}}(n=5)$ mice. Baseline mechanical $(\boldsymbol{A})$ and heat (B; latency) thresholds did not differ in WT and TrkB ${ }^{\mathrm{F} 616 \mathrm{~A}}$ mice, and in both groups mechanical and heat thresholds dropped after CFA injection. However, during the first 60 min after 1NM-PP1, we observed a partial normalization of the mechanical threshold $(\boldsymbol{A})$ and an almost complete normalization of the heat threshold $(\boldsymbol{B})$. These effects disappeared by $90 \mathrm{~min}$ after 1NM-PP1, at which point there were no longer significant differences in mechanical or heat threshold of the CFA-injected hindpaws between WT and $T r k B^{\mathrm{F} 616 \mathrm{~A}}$ mice, but the latter were significantly lower than the thresholds of the paw contralateral to the CFA injection in $\operatorname{TrkB}^{\mathrm{F} 616 \mathrm{~A}}$ mice ${ }^{*} p<0.05$; $\left.{ }^{* * *} p<0.001\right)$. ipsi, Ipsilateral; contra, contralateral.

innocuous tactile stimuli can evoke a withdrawal response, a phenomenon termed mechanical allodynia. Here we studied the effect of 1NM-PP1 on the mechanical hypersensitivity produced in the SNI model of neuropathic pain (Shields et al., 2003). In this model, two of the three branches of the sciatic nerve are ligated, leaving the tibial nerve intact. Within $24 \mathrm{~h}$ of surgery, there is a profound mechanical hypersensitivity of the partially denervated hindpaw. In these experiments, we added 1NM-PP1 to the drinking water $2 \mathrm{~d}$ before the surgical denervation and continued this treatment for $3 \mathrm{~d}$ after the surgery. We tested the mice $3 \mathrm{~d}$ after the surgery and found a profound mechanical allodynia in the control group of mice that drank the 1NM-PP1-treated water. In contrast, the magnitude of the allodynia in the $\operatorname{Trk} \mathrm{B}^{\mathrm{F} 616 \mathrm{~A}}$ mice 
A

von Frey test

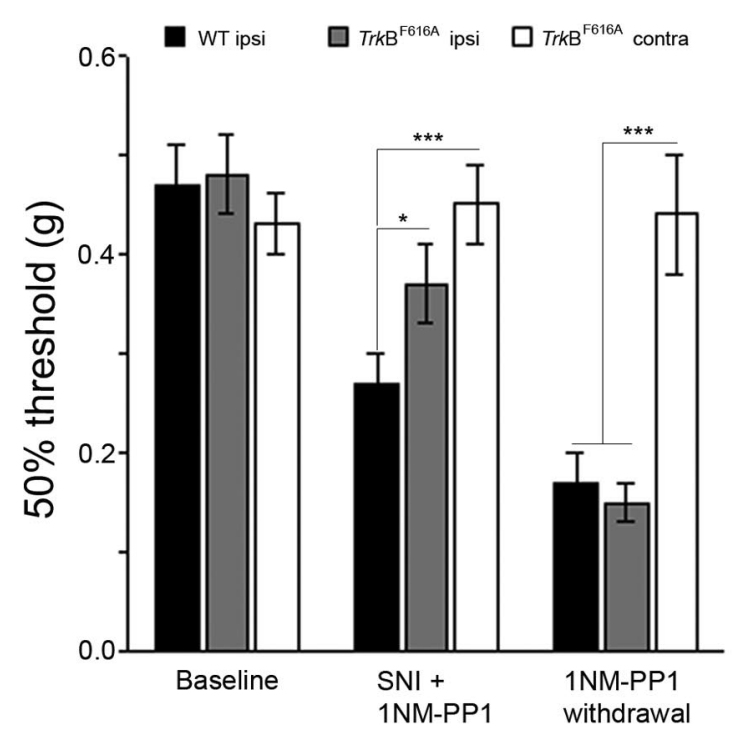

B

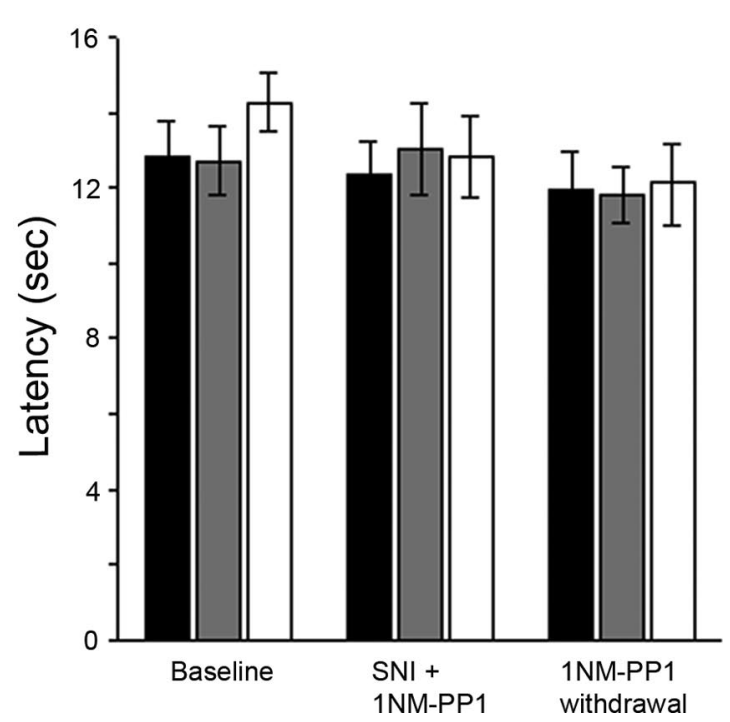

Figure 4. Inhibition of TrkB signaling prevents nerve injury-induced mechanical hyperalgesia. 1NM-PP1 was added to the drinking water (final concentration, $5.0 \mu \mathrm{M}$ ), and mice had unlimited access to the water for $3 \mathrm{~d}$ before they underwent partial SNI. Baseline mechanical $(\boldsymbol{A})$ and heat $(\boldsymbol{B})$ thresholds before SNI did not differ in WT $(n=5)$ and $\operatorname{TrkB}^{\mathrm{F} 616 \mathrm{~A}}(n=5)$ mice. Three days after nerve injury, there was a significant drop of mechanical threshold $(\boldsymbol{A})$ in WT mice compared with $\operatorname{TrkB}{ }^{\mathrm{F} 616 \mathrm{~A}}$ mice $\left.{ }^{*} p<0.05\right)$ or to the contralateral paw of the ${ }^{*}{ }^{\mathrm{FB}}{ }^{\mathrm{F} 616 \mathrm{~A}}$ mice $\left({ }^{* * *} p<0.001\right)$. In neither group of mice was there a change in heat withdrawal threshold after SNI (B). ipsi, Ipsilateral; contra, contralateral.

was significantly reduced. Two days after removal of the 1NMPP1 from the drinking water, the mechanical allodynia reappeared in the TrkB ${ }^{\mathrm{F} 616 \mathrm{~A}}$ mice (Fig. $4 \mathrm{~A}$ ). Despite the profound mechanical allodynia observed in this model, there is limited or no change in heat thresholds. We confirmed this in the present study and further showed that 1NM-PP1 treatment did not influence the sensitivity of the nerve injury model in test of heat sensitivity (Fig. 4B).

These data indicate that $\operatorname{TrkB}$ signaling is not only required for the induction of nerve-injury induced mechanical hypersensitivity but also for its persistence. To provide additional evidence in
A von Frey test

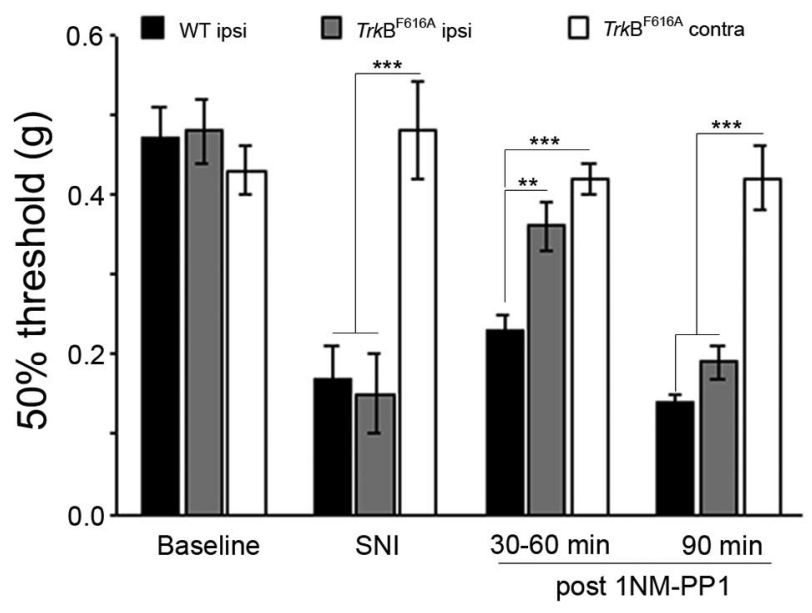

B

Radiant heat

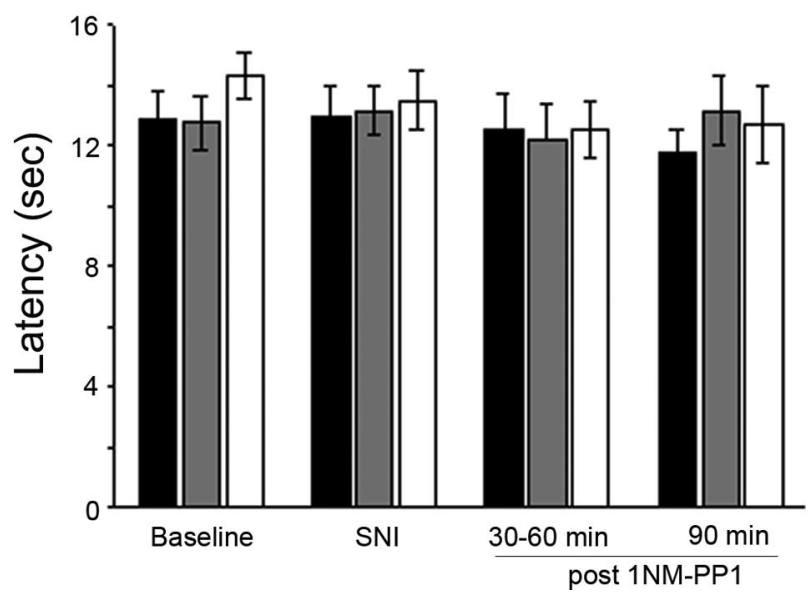

Figure 5. Inhibition of TrkB signaling can reverse already established nerve injury-induced mechanical hyperalgesia. Partial SNI in both WT $(n=5)$ and $\operatorname{TrkB}^{\mathrm{F} 616 \mathrm{~A}}(n=5)$ produced a significant drop of the mechanical withdrawal threshold $(\boldsymbol{A})$ compared with baseline. Injection of 1NM-PP1 (16.6 ng/g, i.p.) 3 d after the SNI reversed the mechanical allodynia in $T_{r k B}{ }^{\mathrm{F} 16 \mathrm{~A}}$ but not in WT mice. The mechanical threshold in WT mice was significantly lower than in TrkB ${ }^{\mathrm{F} 616 \mathrm{~A}}$ mice after 1NM-PP1 ( $\left.{ }^{* *} p<0.01\right)$ and lower than that in the uninjured contralateral paw of the $T r k B{ }^{\mathrm{F} 616 \mathrm{~A}}$ mice $\left({ }^{* * *} p<0.001\right)$. The reversal lasted from 30 to 60 min, reappearing within 90 min of the 1NM-PP1 injection, at which point mechanical thresholds in WT and TrkB ${ }^{\mathrm{F} 616 \mathrm{~A}}$ mice did not differ and both were significantly lower than in the uninjured paw of the TrkB ${ }^{\mathrm{F} 616 \mathrm{~A}}$ mice. ${ }^{* * *} p<0.001$. In neither group did SNI produce a change of the withdrawal threshold to noxious heat $(\boldsymbol{B})$, and 1NM-PP1 injection was without effect in test of heat pain sensitivity. ipsi, Ipsilateral; contra, contralateral.

support of this conclusion, we next asked whether inhibition of TrkB signaling in animals already rendered mechanically hypersensitive could reverse the mechanical hyperalgesia. To this end, we administered 1NM-PP1 (16.6 ng/g) $3 \mathrm{~d}$ after the SNI procedure and evaluated mechanical nociceptive thresholds. Figure $5 \mathrm{~A}$ shows that posttreatment with $1 \mathrm{NM}-\mathrm{PP} 1$ can partially reverse SNI-induce mechanical hyperalgesia in $\operatorname{TrkB}{ }^{\mathrm{F} 616 \mathrm{~A}}$ mice. The duration of the effect of $1 \mathrm{NM}-\mathrm{PP} 1$ was comparable with that observed after inflammation, i.e., the reversal of the mechanical hyperalgesia was transient (lasting no more than $90 \mathrm{~min}$ ). As expected, we found that an intraperitoneal injection of 1NM-PP1 had no effect on heat thresholds in wild-type mice or in $\operatorname{TrkB}{ }^{\text {F616A }}$ mice that did not drink water to which 1NM-PP1 was added (Fig. $5 B)$. Together, these results demonstrate that TrkB phosphoryla- 
A

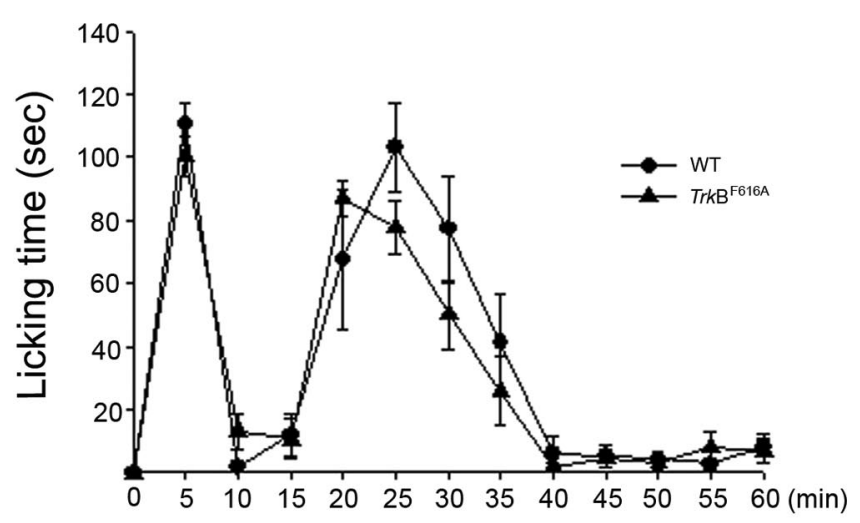

B

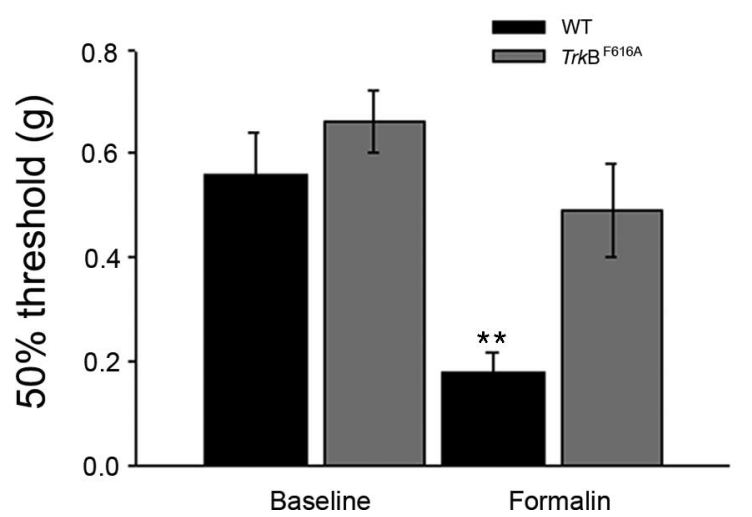

Figure 6. Inhibition of TrkB signaling does not alter formalin-evoked pain behaviors but does prevent postformalin mechanical hypersensitivity. $A$, Wild-type $(n=5)$ and $\operatorname{TrkB}^{\mathrm{F} 616 \mathrm{~A}}$ $(n=5)$ mice drank from 1NM-PP1-treated water for $2 \mathrm{~d}$ before injection of $2 \%$ formalin into the hindpaw. There was no difference in formalin-evoked licking of the hindpaw during either the first or the second phase of pain behavior. $\boldsymbol{B}$, Baseline mechanical thresholds before formalin injection did not differ in WT and TrkB ${ }^{\text {F616A }}$ mice. However, formalin induced a significant decrease of mechanical threshold 90 min after the formalin injection in WT but not in TrkB ${ }^{\text {F616A }}$ that were treated with 1NM-PP1. ${ }^{* *} p<0.01$.

tion is an important contributor and indeed may be required for both the induction and maintenance of nerve injury-induced mechanical hypersensitivity.

TrkB signaling contributes to the state of hypersensitivity but not the immediate pain behavior produced by hindpaw injection of formalin

Intraplantar injection of formalin in mice produces a stereotyped pattern of pain behavior consisting of two distinct phases (Taylor et al., 1997; Shields et al., 2007). Because this model is sensitive to nonsteroidal anti-inflammatory drugs and opiates, the formalin test is generally considered to be a useful model of postoperative pain. The second phase reportedly results from input processed by a spinal cord that was sensitized after the intense activity generated after the injection of formalin. Moreover, reducing BDNF levels decreased pain behavior in the second phase of the formalin test (Kerr et al., 1999; Zhao et al., 2006). Other studies questioned the importance of central sensitization to the second phase of pain behavior (Taylor et al., 1997; Hösl et al., 2006). Here we tested the effect of oral administration of 1NM-PP1 for $2 \mathrm{~d}$ before formalin injection. As illustrated in Figure 6, neither the magnitude nor the time course of the pain behavior in either phase of pain behavior in the formalin test was altered by the 1NM-PP1 pretreatment. These results suggest that either central sensitization is not required for the establishment of the second phase of pain behavior in the formalin test or mechanisms other than TrkB signaling are critical.

However, as we reported previously, the relatively brief duration $(\sim 1 \mathrm{~h})$ of pain behavior produced by formalin is followed by a prolonged period of mechanical hypersensitivity. In contrast to the lack of effect of inhibiting TrkB signaling on the overt pain behavior in the formalin test, we found that oral 1NM-PP1 significantly reduced the postformalin mechanical hypersensitivity. These results distinguish the mechanisms underlying pain behavior associated with different features of the formalin test.

\section{Discussion}

A recent comprehensive review of the contribution of BDNF to the processing of pain messages emphasized the remarkable lack of consensus in the literature (Merighi et al., 2008a). Indeed both pronociceptive and antinociceptive effects of interfering with BDNF-TrkB signaling have been reported. The disagreement likely reflects the varied methods that have been used to alter BDNF or TrkB levels, the fact that selective regulation of these molecules is difficult in the adult and the extent to which downregulation of TrkB signaling was documented. Here we took a chemical-genetic approach, one that directly targeted the phosphorylation step that is initiated when a TrkB ligand binds the receptor. We not only demonstrated profound behavioral effects of treating the $\operatorname{TrkB}{ }^{\mathrm{F} 616 \mathrm{~A}}$ mice with 1NM-PP1 but also confirmed that these changes correlate with inhibition of TrkB phosphorylation. We believe that this approach provides the most direct test of the contribution of TrkB signaling to pain processing, particularly to the thermal and mechanical hypersensitivity that arises in the setting of tissue or nerve injury. Most importantly, because we could reversibly block TrkB phosphorylation in the adult, we conclude that TrkB signaling contributes to both the development of injury-induced persistent pain and its maintenance.

The results were very consistent and robust. Blocking TrkB signaling in the TrkB ${ }^{\mathrm{F} 616 \mathrm{~A}}$ mice, by oral administration of $1 \mathrm{NM}$ PP1, significantly prevented the development of tissue and nerve injury-induced persistent pain. This was true for both heat and mechanical hypersensitivity in the case of CFA-induced inflammation and for the mechanical allodynia produced after partial sciatic nerve injury. In contrast, interfering with TrkB signaling did not alter acute nociceptive processing, as measured by baseline heat or mechanical thresholds, or by the pain behaviors induced by hindpaw injections of capsaicin or formalin. However, 1NM-PP1 treatment in the $\operatorname{TrkB}{ }^{\mathrm{F} 616 \mathrm{~A}}$ mice prevented the mechanical hypersensitivity induced after capsaicin or formalin injection. The simplest explanation for these actions is that TrkB signaling is required for the development of the central sensitization process that underlies the development of persistent heat and mechanical hypersensitivity in the setting of tissue or injury. The fact that we found comparable effects on heat and mechanical hypersensitivity, and in both tissue and nerve injury models, suggests that a common TrkB signaling pathway contributes to the persistent pain triggered by these very different injury conditions. Our results also suggest that the contribution of BDNF to injury-induced central sensitization is via its interaction with TrkB, rather than $\mathrm{p} 75$, a receptor through which BDNF can also signal (Huang and Reichardt, 2003; Fan et al., 2008). Furthermore, although NT-4 also signals via TrkB, the bulk of the evidence points to BDNF, rather than NT-4, as the key TrkB ligand (Heppenstall and Lewin, 2001; Yajima et al., 2002).

Of particular interest to the clinical problem of reversing on- 
going states of injury-induced persistent pain is our finding that blocking TrkB signaling by intraperitoneal injection of 1NM-PP1 after the hypersensitivity state was established significantly reduced the magnitude of the hyperalgesia. The reversal was relatively brief, presumably because of the rapid metabolism of the $1 \mathrm{NM}-\mathrm{PP} 1$, but the result illustrates that maintenance of the persistent pain requires long-term phosphorylation of TrkB. A similar conclusion followed from studies that implicated release of BDNF from activated microglia in the setting of peripheral nerve injury (Coull et al., 2005). Finally, we found that sustaining the blockade of CFA-induced persistent pain (hypersensitivity) also continued presence of 1NM-PP1. When we removed 1NM-PP1 from the drinking water, the mechanical and heat hypersensitivity appeared, indicating that initiation of these hyperalgesic states was delayed and not completely prevented.

That TrkB signaling must be ongoing to sustain the injury induced-pain state was unexpected but interestingly is consistent with two recent studies (Bekinschtein et al., 2007, 2008) that reported that stabilization of long-term memory requires a BDNF-dependent late phase of protein synthesis. Whether the requirement for an ongoing contribution of TrkB phosphorylation to sustain persistent pain reflects continued input from the site of injury or whether it reflects activation of a BNDF source downstream of the afferent drive (e.g., microglia; see below) remains to be determined, but it does suggest that the process of central sensitization can be reversed once established.

Because we administered the 1NM-PP1 by systemic routes (either oral or intraperitoneal), we cannot identify the particular TrkB target in which phosphorylation was abrogated. We favor the view that the relevant TrkB is in the primary afferent nociceptor or in the postsynaptic neurons targeted by the nociceptor, both of which have been shown to express full-length TrkB receptor (Salio et al., 2005; Merighi et al., 2008a). It is also possible that injury-induced de novo expression of $\mathrm{TrkB}$ in nonnociceptive afferents (Mannion et al., 1999; Michael et al., 1999), which in turn engage pain transmission circuitry, is critical. Consistent with the view that the spinal cord or primary afferent is the locus of the relevant $\operatorname{TrkB}$, the great majority of studies that implicated BNDF or TrkB in the development of persistent pain states targeted the spinal cord. Most relevant, perhaps, is that we found that capsaicin-induced secondary hyperalgesia was associated with significant TrkB phosphorylation in the lumbar spinal cord and that prevention of the mechanical hyperalgesia by 1NM-PP1 blocked the TrkB phosphorylation. Of course, systemic administration of $1 \mathrm{NM}-\mathrm{PP} 1$ will concurrently interfere with the enhanced TrkB phosphorylation that occurs in the rostral ventral medulla after tissue injury and which has also been strongly implicated in the facilitation of nociceptive processing at the level of the spinal cord (Guo et al., 2006) (see also Quintão et al., 2008).

As noted above, although 1NM-PP1 blocked the mechanical hyperalgesia produced after an injection of capsaicin or formalin, the immediate pain behavior provoked by these compounds was not altered. The latter result is consistent with the conclusions that TrkB signaling is not required for acute pain responsiveness to a variety of noxious stimuli (heat, mechanical and chemical). However, because it is generally believed that pain behavior in the second phase of the formalin test is strongly influenced by central sensitization of dorsal horn neurons after the intense burst of activity that occurs during the first phase, it was somewhat surprising that abrogation of TrkB signaling was completely without effect on pain behavior during phase 2. This result, however, is consistent with studies from our own laboratory, which argued that pain behavior during the second phase depends as much on afferent drive that occurs during phase 2 as it does on central sensitization produced by activity during the first phase. In our studies, we showed that selective blockade of pain behavior during phase one, with the potent, but short-acting opioid agonist remifentanil, did not reduce pain behavior in the second phase (Taylor et al., 1997; Taylor and Basbaum, 2000). The results of the present study are of particular interest in light of our finding that a mutation of CaMKII completely blocked pain behavior in phase 2 of the formalin test, without influencing formalin mechanical hypersensitivity and without an effect in other persistent pain models (Zeitz et al., 2004). Together with the present results, we conclude that central sensitization is not a critical contributor to pain behavior in the second phase of the formalin test. The effect of CaMKII modification, which can occur in nociceptors as well as in spinal cord "pain" transmission circuits, perhaps reflected disruption of afferent drive during phase 2 .

In summary, using a genetic approach to selectively abrogate TrkB signaling in mice, we provide evidence that signaling via TrkB is a major contributor to injury-induced heat and mechanical hypersensitivity. This is true for both tissue and nerve injuryinduced persistent pain. Acute pain behavior was not altered. Although we cannot identify the particular neurons in which inhibition of TrkB phosphorylation is critical to these effects, the fact that changes produced by both tissue and nerve injury were altered argues that the locus is downstream of the primary afferent fiber. Because we found normal pain behavior in the second phase of the formalin test but decreased postformalin (and postcapsaicin mechanical hyperalgesia), we suggest that disruption of the central sensitization produced by these chemical activators of nociceptors and by the different tissue and nerve injury conditions underlies the profound antinociceptive effects that we observed. Based on these results, we suggest that the critical locus for the TrkB contribution is in the spinal cord dorsal horn. Finally, because interfering with TrkB signaling before the injury prevented development of the persistent pain state and that interfering with TrkB signaling after the injury normalized heat and mechanical withdrawal thresholds, we conclude that TrkB signaling contributes to both the induction and maintenance of injuryinduced persistent pain. Whether the same locus is involved in the induction and maintenance of the persistent pain state remains to be determined.

\section{References}

Bekinschtein P, Cammarota M, Igaz LM, Bevilaqua LR, Izquierdo I, Medina JH (2007) Persistence of long-term memory storage requires a late protein synthesis- and BDNF-dependent phase in the hippocampus. Neuron 53:261-277.

Bekinschtein P, Cammarota M, Katche C, Slipczuk L, Rossato JI, Goldin A, Izquierdo I, Medina JH (2008) BDNF is essential to promote persistence of long-term memory storage. Proc Natl Acad Sci U S A 105:2711-2716.

Chen X, Ye H, Kuruvilla R, Ramanan N, Scangos KW, Zhang C, Johnson NM, England PM, Shokat KM, Ginty DD (2005) A chemical-genetic approach to studying neurotrophin signaling. Neuron 46:13-21.

Cho HJ, Kim JK, Park HC, Kim DS, Ha SO, Hong HS (1998) Changes in brain-derived neurotrophic factor immunoreactivity in rat dorsal root ganglia, spinal cord, and gracile nuclei following cut or crush injuries. Exp Neurol 154:224-230.

Coull JA, Beggs S, Boudreau D, Boivin D, Tsuda M, Inoue K, Gravel C, Salter MW, De Koninck Y (2005) BDNF from microglia causes the shift in neuronal anion gradient underlying neuropathic pain. Nature 438:1017-1021.

Ernfors P, Rosario CM, Merlio JP, Grant G, Aldskogius H, Persson H (1993) Expression of mRNAs for neurotrophin receptors in the dorsal root ganglion and spinal cord during development and following peripheral or central axotomy. Brain Res Mol Brain Res 17:217-226. 
Fan YJ, Wu LL, Li HY, Wang YJ, Zhou XF (2008) Differential effects of pro-BDNF on sensory neurons after sciatic nerve transection in neonatal rats. Eur J Neurosci 27:2380-2390.

Fukuoka T, Kondo E, Dai Y, Hashimoto N, Noguchi K (2001) Brainderived neurotrophic factor increases in the uninjured dorsal root ganglion neurons in selective spinal nerve ligation model. J Neurosci 21:4891-4900.

Groth R, Aanonsen L (2002) Spinal brain-derived neurotrophic factor (BDNF) produces hyperalgesia in normal mice while antisense directed against either BDNF or TrkB, prevent inflammation-induced hyperalgesia. Pain 100:171-181.

Guo W, Robbins MT, Wei F, Zou S, Dubner R, Ren K (2006) Supraspinal brain-derived neurotrophic factor signaling: a novel mechanism for descending pain facilitation. J Neurosci 26:126-137.

Heppenstall PA, Lewin GR (2001) BDNF but not NT-4 is required for normal flexion reflex plasticity and function. Proc Natl Acad Sci U S A 98:8107-8112.

Hösl K, Reinold H, Harvey RJ, Müller U, Narumiya S, Zeilhofer HU (2006) Spinal prostaglandin E receptors of the EP2 subtype and the glycine receptor alpha3 subunit, which mediate central inflammatory hyperalgesia, do not contribute to pain after peripheral nerve injury or formalin injection. Pain 126:46-53.

Huang EJ, Reichardt LF (2003) Trk receptors: roles in neuronal signal transduction. Annu Rev Biochem 72:609-642.

Jimenez-Andrade JM, Martin CD, Koewler NJ, Freeman KT, Sullivan LJ, Halvorson KG, Barthold CM, Peters CM, Buus RJ, Ghilardi JR, Lewis JL, Kuskowski MA, Mantyh PW (2007) Nerve growth factor sequestering therapy attenuates non-malignant skeletal pain following fracture. Pain 133:183-196.

Kaneko M, Hanover JL, England PM, Stryker MP (2008) TrkB kinase is required for recovery, but not loss, of cortical responses following monocular deprivation. Nat Neurosci 11:497-504.

Kerr BJ, Bradbury EJ, Bennett DL, Trivedi PM, Dassan P, French J, Shelton DB, McMahon SB, Thompson SW (1999) Brain-derived neurotrophic factor modulates nociceptive sensory inputs and NMDA-evoked responses in the rat spinal cord. J Neurosci 19:5138-5148

Lever I, Cunningham J, Grist J, Yip PK, Malcangio M (2003) Release of BDNF and GABA in the dorsal horn of neuropathic rats. Eur J Neurosci 18:1169-1174.

Lindsay RM (1996) Role of neurotrophins and Trk receptors in the development and maintenance of sensory neurons: an overview. Philos Trans R Soc Lond B Biol Sci 351:365-373.

Mannion RJ, Costigan M, Decosterd I, Amaya F, Ma QP, Holstege JC, Ji RR, Acheson A, Lindsay RM, Wilkinson GA, Woolf CJ (1999) Neurotrophins: peripherally and centrally acting modulators of tactile stimulusinduced inflammatory pain hypersensitivity. Proc Natl Acad Sci U S A 96:9385-9390.

Marcol W, Kotulska K, Larysz-Brysz M, Kowalik JL (2007) BDNF contributes to animal model neuropathic pain after peripheral nerve transection. Neurosurg Rev 30:235-243; discussion 243.

Merighi A, Bardoni R, Salio C, Lossi L, Ferrini F, Prandini M, Zonta M, Gustincich S, Carmignoto G (2008a) Presynaptic functional TrkB receptors mediate the release of excitatory neurotransmitters from primary afferent terminals in lamina II (substantia gelatinosa) of postnatal rat spinal cord. Dev Neurobiol 68:457-475.

Merighi A, Salio C, Ghirri A, Lossi L, Ferrini F, Betelli C, Bardoni R (2008b) BDNF as a pain modulator. Prog Neurobiol 85:297-2317.

Michael GJ, Averill S, Nitkunan A, Rattray M, Bennett DL, Yan Q, Priestley JV
(1997) Nerve growth factor treatment increases brain-derived neurotrophic factor selectively in TrkA-expressing dorsal root ganglion cells and in their central terminations within the spinal cord. J Neurosci 17:8476-8490.

Michael GJ, Averill S, Shortland PJ, Yan Q, Priestley JV (1999) Axotomy results in major changes in BDNF expression by dorsal root ganglion cells: BDNF expression in large TrkB and TrkC cells, in pericellular baskets, and in projections to deep dorsal horn and dorsal column nuclei. Eur J Neurosci 11:3539-3551.

Pezet S, Malcangio M, McMahon SB (2002) BDNF: a neuromodulator in nociceptive pathways? Brain Res Brain Res Rev 40:240-249.

Quintão NL, Santos AR, Campos MM, Calixto JB (2008) The role of neurotrophic factors in genesis and maintenance of mechanical hypernociception after brachial plexus avulsion in mice. Pain 136:125-133.

Salio C, Lossi L, Ferrini F, Merighi A (2005) Ultrastructural evidence for a pre- and postsynaptic localization of full-length TrkB receptors in substantia gelatinosa (lamina II) of rat and mouse spinal cord. Eur J Neurosci 22:1951-1966.

Shields SD, Eckert WA 3rd, Basbaum AI (2003) Spared nerve injury model of neuropathic pain in the mouse: a behavioral and anatomic analysis. J Pain 4:465-470.

Shields SD, Mazario J, Skinner K, Basbaum AI (2007) Anatomical and functional analysis of aquaporin 1, a water channel in primary afferent neurons. Pain 131:8-20.

Sun RQ, Tu YJ, Yan JY, Willis WD (2006) Activation of protein kinase B/Akt signaling pathway contributes to mechanical hypersensitivity induced by capsaicin. Pain 120:86-96.

Taylor BK, Basbaum AI (2000) Early antinociception delays edema but does not reduce the magnitude of persistent pain in the formalin test. J Pain $1: 218-228$.

Taylor BK, Peterson MA, Basbaum AI (1997) Early nociceptive events influence the temporal profile, but not the magnitude, of the tonic response to subcutaneous formalin: effects with remifentanil. J Pharmacol Exp Ther 280:876-883.

Walker SM, Meredith-Middleton J, Lickiss T, Moss A, Fitzgerald M (2007) Primary and secondary hyperalgesia can be differentiated by postnatal age and ERK activation in the spinal dorsal horn of the rat pup. Pain 128:157-168.

Wang H, Shimizu E, Tang YP, Cho M, Kyin M, Zuo W, Robinson DA, Alaimo PJ, Zhang C, Morimoto H, Zhuo M, Feng R, Shokat KM, Tsien JZ (2003) Inducible protein knockout reveals temporal requirement of CaMKII reactivation for memory consolidation in the brain. Proc Natl Acad Sci U S A 100:4287-4292.

Yajima Y, Narita M, Narita M, Matsumoto N, Suzuki T (2002) Involvement of a spinal brain-derived neurotrophic factor/full-length TrkB pathway in the development of nerve injury-induced thermal hyperalgesia in mice. Brain Res 958:338-346.

Zeitz KP, Giese KP, Silva AJ, Basbaum AI (2004) The contribution of autophosphorylated alpha-calcium-calmodulin kinase II to injury-induced persistent pain. Neuroscience 128:889-898.

Zhao J, Seereeram A, Nassar MA, Levato A, Pezet S, Hathaway G, MorenillaPalao C, Stirling C, Fitzgerald M, McMahon SB, Rios M, Wood JN (2006) Nociceptor-derived brain-derived neurotrophic factor regulates acute and inflammatory but not neuropathic pain. Mol Cell Neurosci 31:539-548.

Zhou XF, Chie ET, Deng YS, Zhong JH, Xue Q, Rush RA, Xian CJ (1999) Injured primary sensory neurons switch phenotype for brain-derived neurotrophic factor in the rat. Neuroscience 92:841-853. 